\title{
Expression of Cyclooxygenase-2 and Ki-67 in Actinic Keratosis and Cutaneous Squamous Cell Carcinoma in Dogs
}

\author{
Sabrina Dos Santos Costa Poggiani ${ }^{1}$, Mário Roberto Hatayde ${ }^{2}$, Renée Laufer-Amorim ${ }^{3}$, \\ Juliana Werner ${ }^{4}$ \\ ${ }^{1}$ Department of Veterinary Medicine, Pioneer Union of Social Integration, Brasilia, Brazil \\ ${ }^{2}$ Department of Veterinary Medicine and Surgery, School of Veterinary Medicine and Animal Science, \\ Sao Paulo State University, Jaboticabal, Brazil \\ ${ }^{3}$ Department of Veterinary Clinic, School of Veterinary Medicine and Animal Science, \\ Sao Paulo State University, Botucatu, Brazil \\ ${ }^{4}$ Werner \& Werner Laboratory, Curitiba, Brazil \\ Email: bina1304@gmail.com, bina1304@hotmail.com
}

Received February 4, 2012; revised March 7, 2012; accepted March 15, 2012

\begin{abstract}
Actinic keratosis is a common disease in humans, which also affects dogs. Lesions occur in chronically sun exposed areas, such as flank, ventral and lateral abdomen. It has been reported that actinic keratosis is a pre-neoplastic disease which may evolve into squamous cell carcinoma (SCC), which is one of the most frequent malignant neoplasm in dogs. The aim of this research was to investigate the relationship between COX-2 and cell proliferation on the outcome of dogs with actinic keratosis and cutaneous squamous cell carcinoma. This study included 10 skin sections of actinic keratosis (G1) and 10 cutaneous SCC (G2). Data including age, breed, gender and histopathological findings were documented. Paraffin-embedded tissues were retrieved for COX-2 and Ki-67 staining. American Pit Bull Terrier dogs were the most affected ones in both G1 and G2, the mean age was $4.3( \pm 0.8)$ years and $5.6( \pm 1.7)$ years, respectively. Mean score of COX-2 immunostaining in G1 and G2 was $8.16( \pm 3.51)$ and $8.56( \pm 1.03)$, respectively. Mean percentage of immunopositive cells for Ki-67 in G1 and G2 was $15.77( \pm 8.81)$ and $17.71( \pm 12.21)$, respectively. There was no association between COX-2 expression and Ki-67, recurrence, survival and metastasis rate $(\mathrm{p}>0.05)$. These findings highlight the role of COX-2 and Ki-67 in carcinogenesis, but do not confirm the relationship between COX-2 expression and increased cell proliferation in dogs. COX-2 may play a role in carcinogenesis, but this pathway is not responsible for cellular proliferation in actinic keratosis and cutaneous SCC in dogs. Both markers were not useful tools to differentiate the outcome of affected dogs.
\end{abstract}

Keywords: Skin; Cancer; Dog

\section{Introduction}

Actinic keratosis is a well documented disease in humans, which also affects dogs and cats [1-3]. Lesions occur in chronically sun exposed areas and mainly in short-coated, white haired skin, such as flank, ventral and lateral abdomen [4]. Actinic keratosis is a pre-neoplastic disease which may evolve into SCC $[1,2,5,6]$. However, it has been reported that actinic keratosis consists on in situ SCC and that there is no biological difference between both, but that in fact, they represent different stage of the same disease $[1,7,8]$. Moreover, it has been reported that there is no clinical distinction between actinic keratosis and SCC [9]. SCC is one of the most frequent malignant neoplasm in dogs, representing the second most common cutaneous neoplasm in this specie. The etiology is not well stabilished in dogs, but UV damage is an important factor $[10,11]$.

Prostaglandins are believed to play a role in the development and progression of some types of cancer [12]. First step in prostaglandins synthesis originated from arachidonic acid is regulated by prostaglandin enzyme $\mathrm{G} / \mathrm{H}$ sintetase, also called cyclooxygenase (COX) [10]. COX-1 modulates physiologic responses such as regulation of renal and vascular homeostasis [13]. COX-2 is usually absent in majority of normal tissues, but can be induced in response to tumor promoters, carcinogens, citocines and growth factors. Upregulation of COX-2 expression has been demonstrated in several neoplasms in dogs, as well as its role in carcinogenesis [14]. COX-2 expression was investigated in 40 samples of SCC in 
dogs, in which it was strongly expressed in neoplastic keratinocytes [10]. Moreover, COX-2 has been prominent in actinic keratosis, SCC and Bowen's disease in humans. It is believed that COX-2 expression due to prolonged sun exposure is followed by increased keratinocytes proliferation. These datas suggest that COX-2 expression is necessary for keratinocytes proliferation and survival after UV radiation [7].

Immunohistochemical analysis of Ki-67 expression has been widely used to evaluate proliferation index and it is a useful tool as a prognostic factor in canine mammary tumors, melanoma and cutaneous mast cell tumor [15-18]. Likewise, downregulation of Ki-67 expression in dogs with mast cell tumor has been related to a higher survival time [19]. Moreover, earlier studies support that staining pattern of Ki-67 in actinic keratosis may separate this entity from others, such as in situ SCC and invasive SCC [20].

The aim of this study was to investigate the association between COX-2 and cellular proliferation on outcome of dogs with actinic keratosis and cutaneous SCC, based on recurrence, metastasis and survival rate. $\mathrm{Ki}-67$ and COX-2 immunorreactivity may aid in better understanding the progression from pre-neoplastic disease (actinic keratosis) to malignant neoplasm (SCC), as well as concerning the correlation beetwen high levels of $\mathrm{COX}-2$ and increased cellular proliferation.

\section{Material and Methods}

Ten skin samples of actinic keratosis and ten skin samples of cutaneous SCC were included in the study. All dogs were attempted at Oncology Service at the Veterinary Teaching of Sao Paulo State University from July 2007 to February 2009 and were included on the basis of the following inclusion criteria: 1) confirmed diagnosis of actinic keratosis or cutaneous SCC; 2) adequate formalin-fixed paraffin embedded material available for histological grade and immunohistochemical staining; 3 ) dog owners written informed consent. Data including age, breed, gender, recurrence, metastasis and survival rate were obtained. Follow-up information was obtained through rechecks and phone calls, every 2 months. Owners were argued about recurrence, concurrent diseases and death.

Diagnosis of actinic keratosis was performed according to Gross et al. (2005). Histological grade of SCC was defined using criteria such as anaplasia (anisocytosis, pleomorphism, anisokaryosis), loss of normal polarity, anarchic growth, atypical mitotic figures and local or vascular invasion. All cases were confirmed as actinic keratosis and SCC by examination of hematoxylin-eosin (HE) sections.

Three-micrometer sections of formalin-fixed paraffinembedded tissue were cut, deparaffinized in xylene, re- hydrated in graded ethanol, and rinsed in distilled water. Immunohistochemical staining was performed using EnVision kit (K4061; EnVision Dual Link System, Peroxidase, DakoCytomation). Antigen retrieval was acessed by three cycles of 5 minutes each in $10 \mathrm{Mm}$ citrate buffer, pH 6.0 in microwave oven. Endogenous peroxidase was quenched by incubating the slides in hydrogen peroxide and methanol (19:1) for 20 minutes. Anti-COX-2 (COX anti-COX-2, monoclonal, clone CX-294, code M3617, DakoCytomation) and anti-Ki-67 (Ki anti-Ki-67, monoclonal, clone MIB-1, code M7240, DakoCytomation) were applied and incubated overnight at $4^{\circ} \mathrm{C}$. Positive control for COX-2 and Ki-67 consisted on kidney and lymph node sections from a clinically healthy dog, respectively. Negative controls were incubated with mouse immunoglobulin (Universal Negative Control-Mouse Primary Antibodies, N1698, DakoCytomation). The reaction was revealed using diaminobenzidine (K3466; Liquid Dab Large Volume Substrate Chromogen System3,3'diaminobenzidine, DakoCytomation) as the chromogen. Sections were counterstained with Mayer's hematoxylin stain and mounted. Only epidermis was considered while evaluating COX-2 expression. The percentage of COX-2 positive cells was graded as: $<5 \% \rightarrow$ $0,5 \%-25 \% \rightarrow 1,26 \%-50 \% \rightarrow 2,51 \%-75 \% \rightarrow 3$ and $>75 \% \rightarrow 4$, and the intensity of COX-2 immunoreactivity was graded as: weak $\rightarrow 1$, moderated $\rightarrow 2$ and strong $\rightarrow 3$. Each COX-2 score represented the product from percentage of positive tumor cells and intensity, as previously described on the literature [14]. A mean value was obtained from 5 counter fields. Ki-67 immunoreactivity was performed on cells (at least 100 cells) containing positive nuclei, at a magnification of $40 \mathrm{x}$ objective. At the end, those results were then converted into percentage.

The Fisher's exact and Tukey's tests were used to compare the frequency of COX-2 and Ki-67 expression in normal and affected skin. Pearson's correlation was performed to describe association between COX-2 and Ki-67 expression. The results underwent Statistical Analysis System (SAS, 9.0. version, SAS Institute, Cary, NC, USA).

\section{Results}

American Pit Bull Terrier dogs were the main breed (5/10) affected by actinic keratosis, followed by Boxer (2/10), Argentine Dogo (1/10), Great Dane (1/10) and Bull Terrier (1/10). American Pit Bull Terrier dogs were also the most common (6/10) ones diagnosed as cutaneous SCC, followed by Argentine Dogo (2/10), Boxer $(1 / 10)$ and Brazilian Terrier (1/10). Mean age of affected dogs by actinic keratosis and cutaneous SCC was 4.3 $( \pm 0.8)$ years and $5.6( \pm 1.7)$ years, respectively. There 
were 7 females and 3 males with actinic keratosis and 6 females and 4 males with cutaneous SCC. All dogs were short-coated and white-haired skin.

Actinic keratosis sections exhibited epidermal changes such as hyperplasia, parakeratosis, orthokeratosis, loss of keratinocytes polarity, moderated cellular pleomorfism and layers disarrangement. Fibroblast proliferation and collagen degeneration were observed in the dermis. Degenerated elastotic fibers revealed solar elastosis. Elastic fibers were disorganized and degenerated. Inflammatory infiltrates was mainly mononuclear. Hair follicles showed infundibular dilatation due to hyperkeratosis. Some cases also exhibited bacterial colonies with neutrophils proliferation.

There were eight well differentiated SCC and two acantholytic SCC. Well differentiated SCC showed epidermal changes such as hyperplasia, hyperkeratosis, parakeratosis, acanthosis and neoplastic keratinocyte proliferation spread over the dermis. Neoplastic cells exhib- ited increased size, moderated nuclear atypia, varying from oval to elongate. Corneal pearls were present in some sections. Perineural invasion was observed in one well differentiated SCC. Lymph vessel invasion by neoplastic cells had been identified in one acantholytic SCC. COX-2 expression showed a diffuse cytoplasmic pattern in keratinocytes, being evident around nuclear membrane. Actinic keratosis samples showed intense staining, over all hyperplastic epidermis. The mean score value was $8.16( \pm 3.51)$. Values above group average were considered "high" and below it, "low". All cutaneous SCC samples showed a high percentage of positive cells, as well as strong COX-2 expression in neoplastic keratinocytes. Mean score value was $8.56( \pm 1.03)$. There was no COX-2 expression in normal skin, neither in negative control. Positive control slide exhibited strong COX-2 expression. There was no difference in COX-2 expression between actinic keratosis and cutaneous SCC ( $\mathrm{p}>$ 0.05) (Tables 1 and 2).

Table 1. Characteristics of specimens from dogs with actinic keratosis.

\begin{tabular}{cccccc}
\hline Dog & Breed & Gender & Age (years) & COX-2 score & Ki-67 (\%) \\
\hline 1 & American Pit Bull & Female & 4 & 12 & 7.4 \\
2 & American Pit Bull & Female & 5 & 8 & 19.54 \\
3 & American Pit Bull & Female & 3 & 8 & 12.86 \\
4 & Great Dane & Female & 4 & 8 & 21.92 \\
5 & Boxer & Male & 6 & 8 & 18.18 \\
6 & American Pit Bull & Female & 4 & 10.4 & 23.88 \\
7 & Argentine Dogo & Male & 4 & 4 & 9.01 \\
8 & American Pit Bull & Female & 4 & 10.4 & 31.84 \\
9 & Boxer & Male & 4 & 0.8 & 3.14 \\
\hline
\end{tabular}

Table 2. Characteristics of specimens from dogs with cutaneous SCC.

\begin{tabular}{|c|c|c|c|c|c|}
\hline Dog & Breed & Gender & Age (years) & COX-2 score & $\mathrm{Ki}-67(\%)$ \\
\hline 1 & Brazilian Terrier & Female & 5 & 9.2 & 13.06 \\
\hline 2 & Argentine Dogo & Female & 5 & 8.4 & 12.91 \\
\hline 3 & American Pit Bull & Female & 4 & 11.2 & 24.09 \\
\hline 4 & American Pit Bull & Female & 4 & 8 & 18.71 \\
\hline 5 & American Pit Bull & Female & 4 & 8 & 12.41 \\
\hline 6 & American Pit Bull & Female & 6 & 8.4 & 19.86 \\
\hline 7 & Boxer & Male & 6 & 8.8 & 48.86 \\
\hline 8 & American Pit Bull & Male & 10 & 7.6 & 13.58 \\
\hline 9 & American Pit Bull & Male & 6 & 8 & 6.45 \\
\hline 10 & Argentine Dogo & Male & 6 & 8 & 7.2 \\
\hline
\end{tabular}


Ki-67 immunostaining was characterized as a nuclear and brownish staining. Actinic keratosis samples exhibited positive cells, especially in keratinocytes on basal and squamous cell layers. In cutaneous SCC samples, neoplastic keratinocytes were immunostaining. Fibroblasts, inflammatory cells, epithelial cells of hair follicles and basal cells of sebaceous glands showed immunoreactivity. There was no difference in Ki-67 expression between actinic keratosis and cutaneous SCC ( $p>0.05)$. Mean percentage of positive cells in normal skin, actinic keratosis and cutaneous SCC samples were 0.4; 15.77 $( \pm 8.81)$ and $17.71( \pm 12.21)$. Values above group average were considered "high" and below it, "low". There was no Ki-67 staining in negative control slide. There was no correlation between COX-2 and Ki-67 expression in both groups $(\mathrm{r}=0.32 ; \mathrm{p}>0.05)$.

One dog presented progression of actinic keratosis into cutaneous SCC, despite the treatment with cryotherapy. Interestingly, this sample showed high Ki-67 expression and low COX-2 expression. Nine dogs showed recurrence of actinic keratosis and seven dogs also presented recurrence of cutaneous SCC. One dog presented one nodule in the cerebrum identified by magnetic resonance, maybe a metastasis of cutaneous SCC. This sample exhibited low Ki-67 and COX-2 expression. One sample showed perineural invasion by neoplastic cells. This specimen showed the highest percentage of positive cells for Ki-67 of the whole group, corresponding to $48.86 \%$.

Survival time after diagnosis was evaluated. All dogs had survival time between 24 to 33 months. One dog with cutaneous SCC has died, due to a chronic hepatitis of unknown etiology, since the owner did not allowed necropsy. On immunohistochemical analysis this sample presented the highest mean score for COX-2 expression, corresponding to 11.2 and $24.09 \%$ of positive cells for Ki-67.

\section{Discussion}

This study is the first to document the expression of COX-2 and Ki-67 in actinic keratosis in dogs, with special interest also on SCC. Mean COX-2 immunostaining score in G1 and G2 was $8.16( \pm 3.51)$ and $8.56( \pm 1.03)$, respectively. Mean percentage of immunopositive cells for $\mathrm{Ki}-67$ in G1 and G2 was $15.77( \pm 8.81)$ and 17.71 $( \pm 12.21)$, respectively. Immunohistochemical analysis showed that there was no difference between COX-2 expression in actinic keratosis and cutaneous SCC. Immunostaining cellular location was similar to others studies, which have shown diffuse immunostaining, being stronger around the nuclear membrane [10]. There was intense expression of COX-2 in actinic keratosis samples, in all hyperplastic epidermis. It is believed that the expression has been induced by excessive and prolonged sun exposure, in agreement to prior findings, which had shown COX-2 overexpression in keratinocytes in vitro exposed to UV light [13]. COX-2 expression impairs epidermal differentiation. The main reasons are the enzyme expression itself, followed by prostaglandins synthesis, which results in epidermal hyperplasia, causing an increased number of cells and hyperkeratosis [21]. COX-2 expression in cutaneous SCC samples was similar to previous studies reported, in which authors observed strong staining in neoplastic keratinocytes in 40 samples of SCC in dogs [10].

Earlier studies demonstrated that COX-2 is induced in SCC [10]. This information provided an interesting model to investigate the role and association of COX-2 expression with other proteins in solar dermatitis. Our results confirm that COX-2 plays a role in development of skin tumors caused by sun exposure, as it has been proposed [22]. It is believed that COX-2 promotes tumor development through angiogenesis. There is an important relationship between COX-2 and mast cells in peritumoral inflammatory infiltrate, which express vascular endothelial growth factor (VEGF) [23]. Besides, the high expression of COX-2 may induce keratinocytes resistance to apoptotic mechanisms. It has been speculated that the mechanisms for which inhibition of apoptosis occur includes the presence of a protein, called nucleobindine, or the intracellular modulation of acid arachidonic [24].

Cellular location and distribution pattern of Ki-67 expression was also in agreement with a report in dogs with melanoma [17]. While keratynocites differentiation occurs towards the corneal layer, these cells have a tendency of losing their nuclei, which justifies the presence of a great number of positive cells on basal and squamous layers. The histologic boundary between actinic keratosis, in situ SCC and invasive SCC is not clear in many cases. Many studies investigate molecules that could be useful in diagnosis. $\mathrm{Ki}-67$ is the most useful one described in human medicine [20]. However, there are no studies in veterinary literature concerning this immunohistochemical differentiation.

COX-2 expression after solar prolonged exhibition was followed by elevation in keratinocytes proliferation [7]. In the present study, there was no correlation between COX-2 expression and cellular proliferation $(\mathrm{r}=$ 0.32). Cellular proliferation induced by COX-2 was considered a result of an impaired epidermal differentiation and did not stimulate proliferation index, which was demonstrated by the downregulation of keratin-10, involucrin and loricrin in suprabasal cells [21]. This is a hypothesis for the absence of correlation between these two markers observed in the present study, but it requires further investigations to be confirmed. Moreover, the lack of association between inflammation and cellular proliferation provides additional evidence that COX-2 
may be a byproduct of carcinogenesis, not its cause. Therefore, both markers should not be used as prognosis tools in dogs affected by actinic keratosis and SCC. Besides, COX-2 expression observed in actinic keratosis and cutaneous SCC specimens supports UV light participation on pathogenesis of both diseases. Finally, results revealed that actinic keratosis has a behavior similar to cutaneous SCC, which suggests that it is not pre-neoplastic disease, but indeed, the initial phase of the SCC. One dog presented progression of actinic keratosis into cutaneous SCC, despite the treatment with cryotherapy. Interestingly, this sample showed high Ki-67 expression and low COX-2 expression, which does not support possible association between inflammation and cellular proliferation reported by the literature [7].

American Pit Bull Terrier dogs were the most affected ones by either actinic keratosis or SCC. This information agrees with previous published studies, which also showed high incidence of actinic keratosis in this dog breed [25]. It is stated that American Pit Bull Terrier dogs have predilection to develop cutaneous SCC [4]. Authors believe the high incidence of actinic keratosis in this breed may be due to this population increasing in last years and also due to the prolonged sun exposure reported by all owners.

Mean age of dogs with actinic keratosis and cutaneous SCC was $4.3( \pm 0.8)$ and $5.6( \pm 1.7)$ years, respectively. It disagrees from other authors, who stated that the disease occurs mainly in older dogs [4]. These data indicate that this population has been exposed to risk factors earlier, especially UV light, leading to the development of the disease in young age.

Histopathological findings in actinic keratosis were similar to the ones described in veterinary literature [4]. Solar elastosis observed on these samples highlights that solar injury plays a role in the etiology of the diseases. Cutaneous SCC samples (8/10) also showed solar elastosis. The absence of this finding in two samples supports the possibility of another etiologic factor, such as papillomavirus, which has been identified in 9/42 samples of canine SCC [26]. It has been reported that acantholytic SCC is an uncommon histological grade and that well differentiated SCC is the most common one in dogs [4]. Our results agree with this information, based on the low frequency of acantholytic SCC.

The incidence of perineural invasion was evaluated and it was observed in only one sample. In this case, clinical evolution was breaf (4 months) and the lesion was located in the escrotum. In a previous study, $69 \%$ of the human patients who presented perineural invasion developed local lymph node metastasis [27]. Another case had lymph vessel invasion by neoplastic cell. Nevertheless, this female did not present lymph nodes metastasis, which disagrees with the predictive value estab- lished by other authors, who showed a positive relationship between invasion of lymphatic vessels and metastasis in breast cancer in women [28].

The main histological finding that differentiates actinic keratosis from cutaneous SCC is the basal membrane invasion by neoplastic cells. However, some authors believe this identification may be difficult in samples in which the basal membrane is not easily delimited, for reasons such as broken section, presence of intense inflammatory infiltrate and small samples [20]. These authors identified different patterns of Ki-67 protein staining in samples of actinic keratosis and cutaneous SCC of human patients, which can be used to differentiate these two diseases when histopathological evaluation is not enough. This data disagrees with this information. The immunoistochemical pattern in actinic keratosis and cutaneous SCC were similar and the percentage of marked cells did not vary in accordance with the histopathologycal diagnosis.

The presence of histopathological changes, associated with clinical presentation and immunoistochemical characteristics show strong similarities between actinic keratosis and cutaneous SCC, which allow us to suggest that both correspond to the same disease, but in different phases, as it already been reported $[1,8]$. The reluctance in changing the terminology of the actinic keratosis is mainly due to the psychological impact that the word "carcinoma" can cause in owners of dogs [4].

Skin diseases caused by prolonged sun exposure have been widely studied in human medicine [8,20,29-32]. However, it is important to emphasize that it does not occur in veterinary medicine, despite of the high incidence of these diseases $[3,10,25,33]$. This study described epidemiological, histopathological and immunohistochemical data in actinic keratosis and cutaneous SCC in dogs. Our results provide new evidence regarding the association between Ki-67 expression and its association with actinic keratosis. Ki-67 expression has no diagnostic or prognostic value in dogs with actinic keratosis. In contrast with our results, it has been observed that the pattern of Ki-67 staining can be used to support differentiation of actinic keratosis, in situ SCC, and squamous cell carcinoma [20]. We believe that COX-2 may play a role in carcinogenesis, but this pathway is not responsible for cellular proliferation in actinic keratosis and cutaneous SCC in dogs.

\section{Acknowledgements}

The authors are thankful to CAPES for the master fellowship granted.

\section{REFERENCES}

[1] M. Lebwohl, "Actinic Keratosis: Epidemiology a Pro- 
gression to Squamous Cell Carcinoma," British Journal of Dermatology, Vol. 149, No. 66, 2003, pp. 31-33. doi:10.1046/j.0366-077X.2003.05621.x

[2] E. Papadogiannakis, V. Kontos and K. Frangia, "A Case of Canine Squamous Cell Carcinoma Secondary to Solar Keratosis (Actinic Carcinoma in Situ)," Journal of the Hellenic Veterinary Medical Society, Vol. 59, No. 1, 2008, pp. 64-70.

[3] C. Favrot, M. Welle, M. Heimann, D. L. Godson and F. Guscetti, "Clinical, Histologic, and Immunohistochemical Analyses of Feline Squamous Cell Carcinoma in Situ," Veterinary Pathology, Vol. 46, No. 1, 2009, pp. 25-33. doi:10.1354/vp.46-1-25

[4] T. L. Gross, P. J. Ihrke, E. J. Walder and V. K. Affolter, "Skin Diseases of the Dog and Cat: Clinical and Histopathologic Diagnosis," Blackwell, Oxford, 2005, p. 932. doi:10.1002/9780470752487

[5] J.-P. Ortonne, "From Actinic Keratosis to Squamous Cell Carcinoma," British Journal of Dermatology, Vol. 146, No. 61, 2002, pp. 20-23. doi:10.1046/j.1365-2133.146.s61.6.x

[6] C. J. Cockerell, "Pathology and Pathobiology of the Actinic (Solar) Keratosis," British Journal of Dermatology, Vol. 149, No. 66, 2003, pp. 34-36. doi:10.1046/j.0366-077X.2003.05625.x

[7] J.-P. Ortonne, "Anti-Inflammatory vs. Inflammatory Treatments for Actinic Keratosis," Journal of Cosmetic Dermatology, Vol. 2, No.3-4, 2003, pp. 135-140. doi:10.1111/j.1473-2130.2004.00093.x

[8] J. Röwert-huber, M. J. Patel, T. Forschner, C. Ulrich, J. eberle, H. Kerl, W. Sterry and E. Stockfleth, "Actinic Keratosis Is an Early in Situ Squamous Cell Carcinoma: A Proposal for Reclassification," British Journal of Dermatology, Vol. 156, No. 3, 2007, pp. 8-12. doi:10.1111/j.1365-2133.2007.07860.x

[9] E. Stockfleth and H. Kerl, "Guidelines for the Management of Actinic Keratosis," European Journal of Dermatology, Vol. 16, No. 6, 2006, pp. 599-606.

[10] E. M. P. Almeida, C. Piché, J. Sirois, M. Doré, "Expression of Cyclo-oxygenase-2 in Naturally Occuring Squamous Cell Carcinomas in Dogs," The Journal of Histochemistry \& Cytochemistry, Vol. 49, No. 7, 2001, pp. 867-875. doi:10.1177/002215540104900707

[11] D. Scott, W. H. Miller and C. E. Griffin, "Small Animal Dermatology," 6th Edition, Saunders, Philadelphia, 2001, p. 1528.

[12] R. L. Howe, "Inflammation and Breast Cancer. Cyclooxygenase/Prostaglandin Signaling and Breast Cancer," Breast Cancer Research, Vol. 9, No. 4, 2007, pp. 210219. doi: $10.1186 /$ bcr1678

[13] S. Y. Buckman, A. Gresham, P. Hale, G. Hruza, J. Anast, J. Masferrer and A. P. Pentland, "COX-2 Expression Is Induced by UVB Exposure in Human Skin: Implications for the Development of Skin Cancer," Carcinogenesis, Vol. 19, No. 5, 1998, pp. 723-729. doi:10.1093/carcin/19.5.723

[14] A. B. de Nardi, C. R. Daleck, C. H. M. Souza, R. L. Amorim, S. Rodaski, C. Calderon and R. Torres, "Cyclo- oxygenase-2 Expression in Mammary Tumors in Dogs and Its Correlation to Histologic and Biologic Behavior," Veterinary and Comparative Oncology, Vol. 3, No. 1, 2005, pp. 56-57. doi:10.1111/j.1476-5810.2005.064ak.X

[15] D. A. P. C. Zuccari, M. V. Pavam, A. C. B. Terzian, R. S. Pereira, C. M. Ruiz and J. A. Andrade, "Immunohistochemical Evaluation of E-cadherin, Ki-67 and PCNA in Canine Mammary Neoplasias: Correlation of Prognostic Factores and Clinical Outcome," Pesquisa Veterinária Brasileira, Vol. 28, No. 4, 2008, pp. 207-215. doi:10.1590/S0100-736X2008000400003

[16] S. Roels, K. Tilmant and R. Ducatelle, "PCNA and Ki67 Prolieration Markers as Criteria for Prediction of Clinical Behaviour of Melanocytic Tumours in Cats and Dogs," Journal of Comparative Pathology, Vol. 121, No. 1, 1999, pp. 13-24. doi:10.1053/jcpa.1998.0291

[17] C. Laprie, J. Abadie, M-F. Amardeilh, J-L. L. E. Net, M. Lagadic and M. Delverdier, "MIB-1 Immunoreactivity Correlates with Biologic Behaviour in Canine Cutaneous Melanoma," Veterinary Dermatology, Vol. 12, No. 3, 2001, pp. 139-147. doi:10.1046/j.1365-3164.2001.00236.x

[18] J. D. Webster, "Evaluation of Prognostic Markers for Canine Mast Cell Tumors Treated with Vinblastine and Prednisone," BMC Veterinary Research, Vol. 4, 2008, p. 32. doi:10.1186/1746-6148-4-32

[19] O. Kiyokazu, Y. Tetsushi, N. Koji and N. Isao, "Prognostic Significance of Surgical Margin, Ki-67 and Cyclin D1 Protein Expression in Grade II Canine Cutaneous Mast Cell Tumor," Journal of Veterinary Medical Science, Vol. 69, No. 11, 2007, pp. 1117-1121. doi:10.1292/jvms.69.1117

[20] C. W. Oh and N. Penneys, "P27 e MIB-1 Expression in Actinic Keratosis, Bowen Disease, and Squamous Cell Carcinoma," The American Journal of Dermatopathology, Vol. 26, No. 1, 2004, pp. 22-26. doi:10.1097/00000372-200402000-00004

[21] G. Neufang, G. Fürstenberger, M. Heidt, F. Marks and K. Müller-Decker, "Abnormal Differentiation of Epidermis in Transgenic Mice Constitutively Expressing Cyclooxygenase-2 in Skin," Proceedings National Academy of Sciences of the United States of America, Heidelberg, 2001, pp. 7629-7634.

[22] F. Naghshvar, Z. Torabizadeh, O. Emadian, A. Khalilian, I. Maleki and K. Enami, "Correlation of Cyclooxygenase 2 (Cox-2) Presentation and Inflammatory Cells Infiltration in Colorectal Cancer: A Histopathological and Immunohistochemical Study," Research Journal of Biological Sciences, Vol. 3, No. 8, 2008, pp. 916-918.

[23] A. Grützkau, S. Krüger-Krasagakes, H. Baumeister, C. Schwarz, H. Kögel, P. Welker, U. Lippert, B. M. Henz and A. Möller, "Synthesis, Storage, and Release of Vascular Endothelial Growth Factor/Vascular Permeability Factor (VEGF/VPF) by Human Mast Cells: Implications for the Biological Significance of $\mathrm{VEGF}_{206}$," Molecular Biology of the Cell, Vol. 9, No. 4, 1998, pp. 875-884.

[24] K. Subbaramaiah, N. Altorki, W. J. Chung, J. R. Mestre, A. Sampat and A. J. Dannenberg, "Inhibition of Cyclooxygenase-2 Gene Expression by p53," Journal of Bio- 
logical Chemistry, Vol. 274, No. 16, 1999, pp. 1091110915. doi:10.1074/jbc.274.16.10911

[25] B. Tammenhain, M. R. Farias, A. Obladen and J. Werner, "Estudo Retrospectivo da Dermatite ActÍnica em Cães da Raça pit Bull Terrier," Proceedings Congresso Brasileiro da Associação Nacional de Clínicos Veterinários de Pequenos Animais, Maceió, 2008, pp. 1-3.

[26] N. Zaugg, G. Nespeca, B. Hauser, M. Ackermann and C. Favrot, "Detection of Novel Papillomaviruses in Canine Mucosal, Cutaneous and in Situ Squamous Cell Carcinoma," Veterinary Dermatology, Vol. 16, No. 5, 2005, pp. 290-298. doi:10.1111/j.1365-3164.2005.00467.x

[27] E. F. Velazquez, G. Avala, H. Liu, A. Chaux, M. Zanotti, J. Torres, S. I. Cho, J. E. Barreto, F. Soares and A. L. Cubilla, "Histologic Grade and Perineural Invasion Are More Important than Tumor Thickness as Predictor of Nodal Metastasis in Penile Squamous Cell Carcinoma Invading 5 to $10 \mathrm{~mm}$," American Journal of Surgical Pathology, Vol. 32, No. 7, 2008, pp. 974-979. doi:10.1097/PAS.0b013e3181641365

[28] V. F. Z. Marinho, K. Metze, F. S. F. Sanches, G. F. S. Rocha and H. Gobbi, "Marcadores Moleculares em câncer de Mama Preditivos de Metástases Axilares," Revista da Associação Médica Brasileira, Vol. 54, No. 3, 2008, pp. 203-207.

[29] P. M. Carpenter, K. G. Linden, C. E. McLaren, K.-T. Li,
S. Arain, R. J. Barr, P. Hite, J. D. Sun and F. L. Meyskens, "Nuclear Morphometry and Molecular Biomarkers of Actinic Keratosis, Sun-Damaged, and Nonexposed Skin," Cancer Epidemiology, Biomarkers \& Prevention, Vol. 13, No. 12, 2004, pp. 1996-2002.

[30] M. Ulrich, A. Maltusch, J. Röwert-huber, S. González, W. Sterry, E. Stockfleth and S. Astner, "Actinic Keratoses: Non-Invasive Diagnosis for Field Cancerisation," British Journal of Dermatology, Vol. 156, No.. 3, 2007, p. 13-17. doi:10.1111/j.1365-2133.2007.07865.x

[31] B. Berman, L. Bienstock, L. Kuritzky, E. J. Mayeaux and S. K. Tyring, "Actinic Keratoses: Sequelae and Treatments," The Journal of Family Practice, Vol. 55, 2006, pp. 1-8.

[32] T. A. Silva, G. Coelho, A. L. Bocca and F. F. Cavalcante Neto, "Expression of Apoptotic, Cell Proliferation Regulatory, and Structural Proteins in Actinic Keratosis and Their Association with Dermal Elastosis," Journal of Cutaneous Pathology, Vol. 34, No. 4, 2006, pp. 315-323. doi:10.1111/j.1600-0560.2006.00621.x

[33] N. Pronovost, M. M. Suter, E. Mueller, J. Sirois and M. Doré, "Expression and Regulation of Cyclooxygenase-2 in Normal and Neoplastic Canine Keratinocytes," Veterinary and Comparative Oncology, Vol. 2, No. 4, 2004, pp. 222-233. doi:10.1111/j.1476-5810.2004.00055.x 